\title{
Quantitative genetic background effects on the Antennapaedia phenotype in Drosophila melanogaster
}

\author{
Debra R. Possidente, \\ Jennifer Greaux and \\ Bernard Possidente*
}

Skidmore College, Department of Biology, Saratoga Springs, NY 12866, U.S.A.

Genetic background variation influencing expression of the Antennapaedia homeotic phenotype was examined by crossing the $A_{n t p}{ }^{73 b}$ allele of the Antennapaedia locus reciprocally into seven isogenically derived wild type strains of Drosophila melanogaster and their genetically heterogeneous parent strain, Dover. The parent Antp ${ }^{73 b}$ strain's Antennapaedia phenotype shows a small patch of untransformed antennal tissue remaining on the homeotic femur. The size of this patch was used as an assay for background variation influencing expression of the Antp ${ }^{73 b}$ homeotic mutation. Patch size varied approximately six-fold across the different genetic backgrounds. Effects of maternal parent, sex and sex-linkage were also observed.

\section{INTRODUCTION}

Homeotic mutations identify genes that play a major role in the developmental control of gene expression (Holland, 1989). Antennapaedia is one of the largest and best understood homeotic complexes, and recent studies have demonstrated the complex temporal and spatial expression of Antennapaedia gene products (Carroll et al., 1986, 1988), as well as regulatory interactions between Antennapaedia and other loci (Carroll, 1988; Hafen, 1984). Any loci capable of altering the quantitive expression of homeotic loci are potentially important for understanding the full range of homeotic gene function, and are particularly important for understanding the evolutionary significance of genetic variation at homeotic loci.

A number of earlier studies demonstrated that both the frequency and severity of homeotic phenotypes could be altered radically by changes in background genotypes (Ouweneel, 1970; Waddington, 1956; Goldschmidt and LedermanKlein, 1958). Other studies in Drosophila have also shown that quantitative genetic variation derived from wild-type populations can support rapid transformations of major discrete phenotypic traits, such as scutellar bristle number (Rendel, 1967) and abnormal abdomen (Templeton, 1982).

\footnotetext{
* To whom correspondence should be addressed.
}

Myashita et al. (1986) have demonstrated that unlinked modifier genes alter the activity levels of several different enzymes in Drosophila, at least in part by affecting levels of gene product. We thought it might be of interest, in light of these studies, to re-examine quantitative genetic effects on a homeotic phenotype. The starting point for this study was our observation that crossing the Antennapaedia allele $A n t p^{73 b}$ into a wild type genetic background from a stock strain altered its homeotic effect very little, in general, except that a small patch of untransformed antennal third segment (AIII) tissue on the proximal femur was greatly increased in size. Stocker et al. (1976) noted a similar increase in an AIII patch when they generated a double heterozygote between two different Antennapaedia alleles. Here we describe quantitative effects of strain, maternal parent, sex and sex-linkage on the degree of transformation of the AIII segment by $A n t p^{73 b}$, using seven isogenically derived wild-type strains of Drosophila melanogaster.

\section{MATERIALS AND METHODS}

\section{Drosophila stocks}

The Antennapaedia strain used in all crosses was $A n t p^{73 b} / T M 3, S b S e r$, obtained from T. Kaufman at the University of Indiana. This mutation shows 


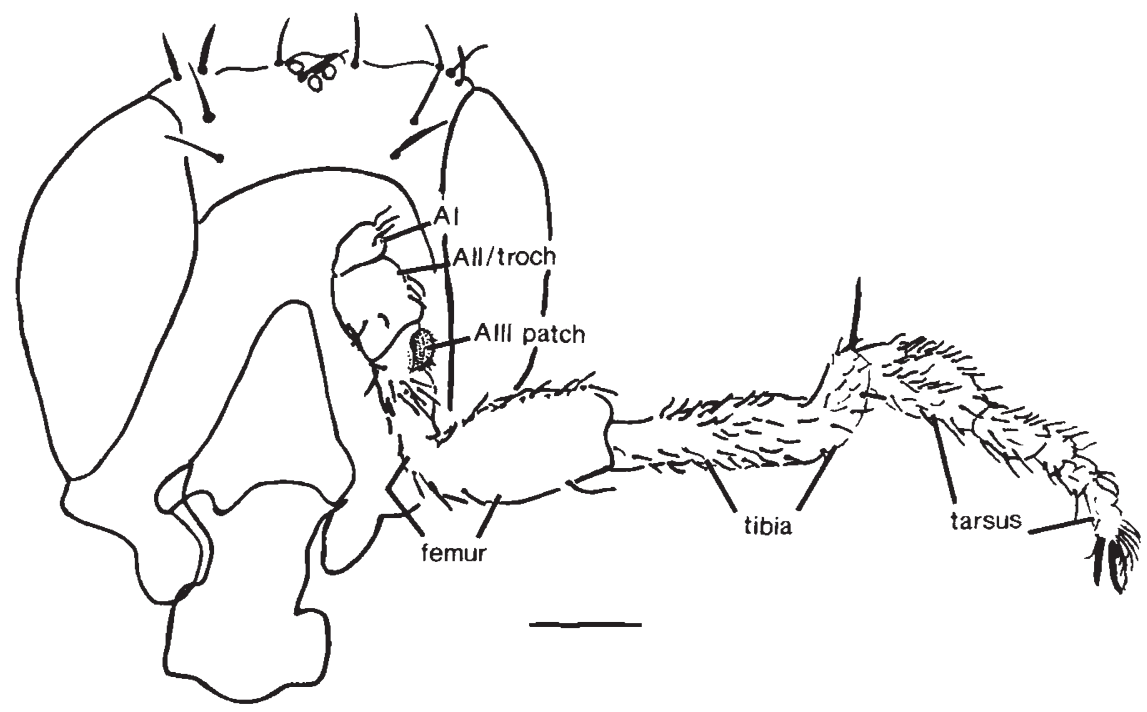

Figure 1 An example of the Antp ${ }^{73 b}$ phenotype. One side of a severely transformed appendage is shown. Note the AIII patch just anterior to a group of campaniform sensilla. Proximal structures are antenna-like, distal structures are leg-like. This male Antp ${ }^{73 b}$ fly arose in the progeny of a Dover female and an $A n t p^{73 b}$ male. Scale bar $200 \mu \mathrm{m}$.

a consistent and severe transformation of antennal structures to leg structures (fig. 1). Antp ${ }^{73 b}$ is dominant, and is caused by an inversion affecting a regulatory region of the Antennapaedia complex (Schneuwly et al., 1987a). The transformation of antennae to legs by this allele appears to be the result of ectopic production of Antennapaedia gene product (Schneuwly et al., 1987b).

This transformation follows the sequential homology described for $A n t p^{R}$ by Postlethwaite and Schneiderman (1971). Proximally, Antp ${ }^{73 b}$ does not appear to transform the first antennal segment (AI) towards coxa, but causes a hybrid second antennal segment (AII) mixed with trochanter structures. Proceeding distally, $A n t p^{73 b}$ radically transforms the third antennal segment (AIII) and arista to leg. Except for a small island of AIII tissue on the proximal femur segment (which will be the focus of these experiments) and a few arista filaments emerging from the fifth tarsal segment, mesothoracic femur and tarsal structures are easily discernible on $A n t p^{73 b}$ heads, on both sides of all flies, though leg tissue may be somewhat telescoped. The presence of claws and the extent of aristae production varies.

We used a sample from the Dover wild-type strain, provided to us by J. D. Mohler, University of Iowa. Seven different isogenically derived lines of Dover flies, and a randomly outbred Dover control population were used to study the phenotype of $A n t p^{73 b}$ expressed against different genetic backgrounds. The seven strains $(7,53,60$, $63,67,71$ and 83 ) were each derived from a single male passed through a balanced lethal marker chromosome breeding scheme designed to make chromosomes I, II and III homozygous. All were derived from the original outbred Dover stock in 1983 and maintained since then by random breeding within lines until they were crossed with the Antp ${ }^{73 b}$ stock in 1988. Each line was maintained in five standard shell vials which were occasionally mixed within lines to supplement individual vials during occasional periods of low viability.

\section{EXPERIMENTAL DESIGN}

Virgin Dover flies from each isogenically derived strain and from outbred Dover flies were crossed reciprocally with $A n t p^{73 b}$ virgins. $A n t p^{73 b}$ flies were also mated inter se. Approximately five males and five females were mated per standard shell vial with Carolina instant fly medium for food, and maintained at a temperature of $25 \pm 1^{\circ} \mathrm{C}$. Five replicate vials were initiated for each cross where the males were $A n t p^{73 b}$ and three replicate vials where the females were $A n t p^{73 b}$. Parents were removed on day six after mating, and progeny began eclosing on day nine. At least 19 flies of each sex for each cross were collected over days eleven and twelve. 
Genetic differences among the strains are indicated by variation in patch size among strains that significantly exceeds variation within strains. Differences between the female progeny of a reciprocal cross indicate maternal effects (ignoring paternal effects) since they have both an Xchromosome and autosome from each parental strain but have different mothers. Differences between male progeny of a reciprocal cross can reflect both maternal effects and sex chromosome effects. Sex chromosome effects can be detected independently of maternal effects by a significant sex by maternal parent interaction within the progeny of a reciprocal cross.

The progeny were divided into two classes, those with $A n t p^{73 b}$ mothers and those with nonAntp ${ }^{73 b}$ mothers, to test for interactions among maternal parent, cross and sex factors. A strain by maternal parent interaction in the reciprocal female progeny across all strains will test for strain effects on maternal effects, a strain by maternal parent by sex interaction will test for strain differences in sex chromosome effects, and a strain by sex interaction will reveal strain differences for effect of sex on AIII patch size.

\section{MOUNTING HEADS FOR VIEWING}

Harvested flies were anaesthetized with ether and decapitated with a razor blade. Heads were grouped on glass slides by cross, sex and vial, mounted in Permount, proboscis upward in the groove between two coverslips, and covered by a third coverslip.

\section{MEASUREMENT OF AIII PATCH SIZES}

Patches of AIII tissue were visible on both transformed antennae on all flies except for seven appendages in the Antp ${ }^{73 b} \times A n t p^{73 b}$ cross. These patches always emerged in the same location: proximally on the femur, in a medial position just anterior to a group of campaniform sensilla, under a shoulder-like overhang of AII tissue (fig. 2).

Placing the coverslip over the proboscis caused it to retract and hold each antenna in a groove between the proboscis and the eye in approximately the same position in all flies. There was some variability in the way the more distal parts of the antennal leg floated to rest under the coverslip, making a variable rotation of the AIII patch under study inevitable. Since the patch has depth as well as width, it presented roughly the same
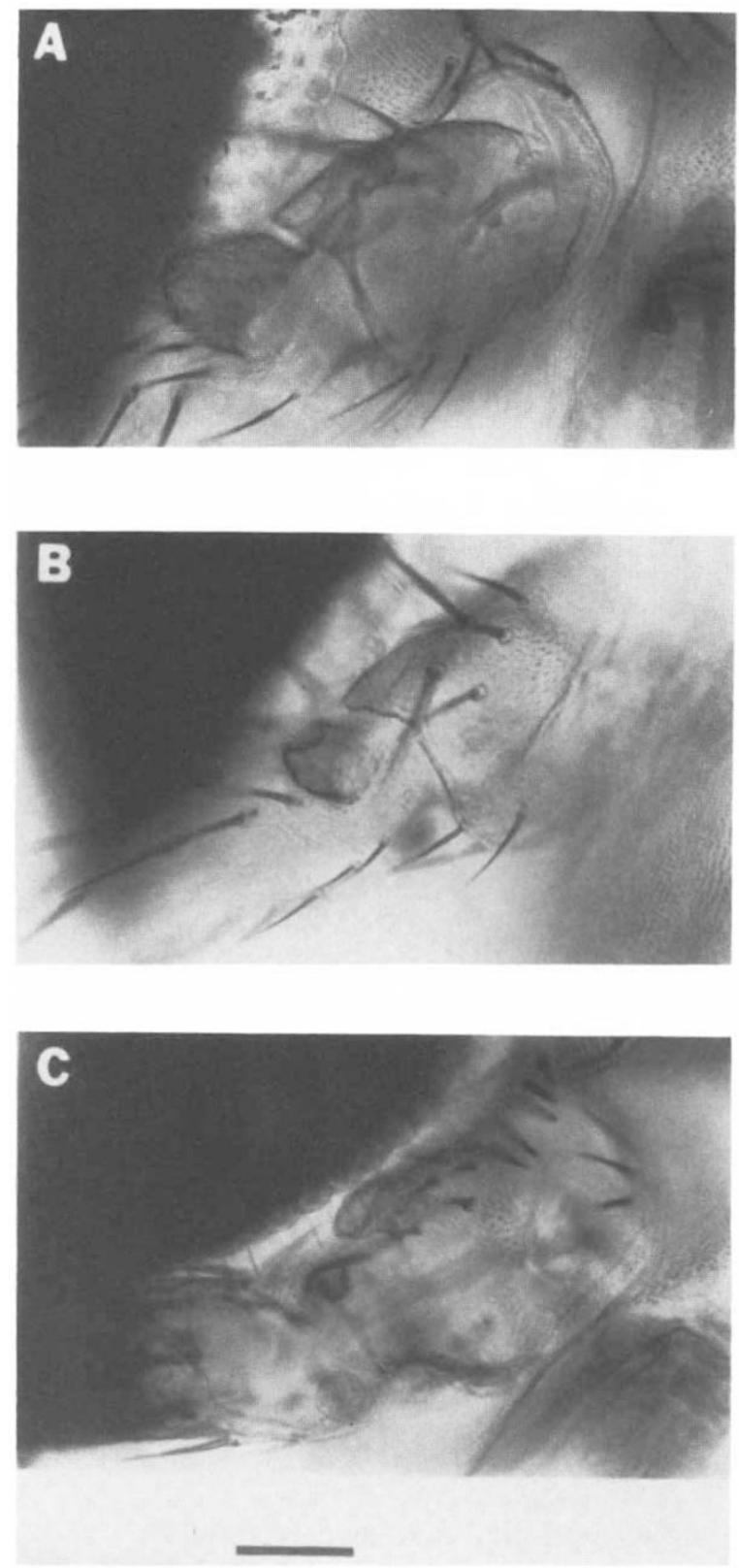

Figure 2 The size range of the AIII patch under study is shown. All three photographs were taken at the same magnification and show females from different crosses. The dark tissue to the left is eye. The AIII patch is found under an overhanging shoulder of AII tissue. A, Progeny of a Dover female and Antp ${ }^{73 b}$ male; B, Progeny of a strain 60 female and Antp ${ }^{73 b}$ male; C, Progeny of an Antp ${ }^{73 b}$ female and $A n t p^{73 b}$ male. Scale bar $55 \mu \mathrm{m}$.

two-dimensional area from the perspective available through the microscope, regardless of the rotation. 
Each patch was viewed under a compound light microscope at $500 \times$ magnification and projected on to a video screen. The video images were traced onto plastic transparencies, which were photocopied without change in size. Appendages where AIII patches were not clearly visible because of air bubbles or leakage of Permount from the preparation were omitted. If only one appendage was measured it was labelled side " 1 "; when two appendages were measured on the same fly, they were labelled side " 1 " and side " 2 " in order of measurement, regardless of left-right anatomical orientation. The photocopies were traced on a digitizing tablet (Jandel Scientific, Corte Madera, CA, U.S.A.). The areas of the irregular shapes were calculated using Sigma-Scan software (Jandel Scientific) and converted to square microns using a 50-micron square calibration image.

\section{DATA ANALYSIS}

The significance of the main effects (strain, sex, maternal parent) and the various interactions was determined by analysis of variance using the GLM procedure of the SAS software package (1982). Genetic and phenotypic correlations between side 1 and side 2 values were determined by analysis of covariance using the SAS NESTED procedure. The number of usable preparations varied from eight to 47 per sex per reciprocal cross. Means are reported \pm their standard errors. All univariate statistical analyses use side "1" measurements only, since the patch on the second appendage was not clearly visible after fixation in about 25 per cent of the preparations.

\section{RESULTS}

The average untransformed AllI patch size for each sex of progeny in each cross is shown in table 1. The average patch area among all the flies measured was $1401 \pm 27$ square micrometres. There was wide variation among the progeny of the different crosses $(F[8,783]=36 \cdot 0, P<0.0001$; fig. $3)$. The $A n t p^{73 b}$ allele crossed into Dover flies showed the largest AIII patch size of $2094 \pm 89$ square microns, and the original Antp ${ }^{73 b}$ strain showed the smallest mean patch size of $299 \pm 28$ square microns. The progeny of the Antp ${ }^{73 b}$ and isogenically derived strains averaged $1392 \pm 82$ square microns overall, and ranged from $1801 \pm$ 103 (strain 63) to $979 \pm 71$ square microns (strain 7).

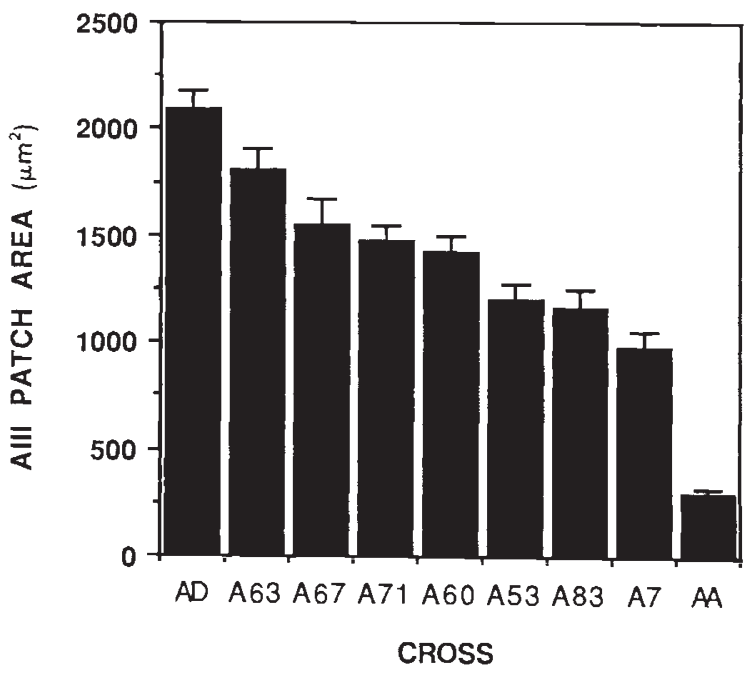

Figure 3 Mean \pm s.e.m. of untransformed AIII patch size for the same $A n t p^{73 b}$ allele against nine different genetic backgrounds: AD is the outbred wild-type Dover background, A63-A7 are the seven isogenically derived backgrounds from the Dover population, and AA is the original Antp $p^{73 b}$ stock. Strain means are pooled across sexes and reciprocal crosses.

The reciprocals did not differ significantly in the outbred Dover $\times A n t p^{73 b}$ cross, but overall there was a larger mean patch size in progeny of $A n t p^{73 b}$ females, by approximately 520 square microns, in the reciprocal crosses between Antp ${ }^{73 b}$

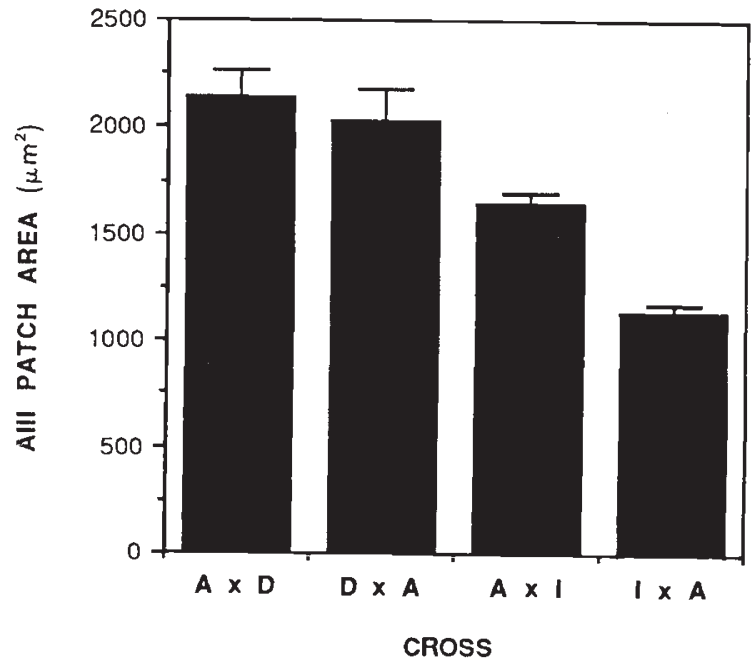

Figure 4 Mean \pm s.e.m. for untransformed AIII patch size for the two reciprocal cross categories for $A n t p^{73 b}$ and the outbred Dover population $(\mathrm{A} \times \mathrm{D}$ and $\mathrm{D} \times \mathrm{A})$ and the $A n t p^{73 b}$ crossed with isogenically derived strains $(\mathrm{A} \times \mathrm{I}$ and $I \times A)$. The reciprocal crosses for the seven isogenically derived strains are pooled for this comparison since all showed a higher mean patch size with $A n t p^{73 b}$ mothers. Means are pooled across sexes. 
Table 1 Mean AIII patch areas (square micrometres) of progeny from Antp ${ }^{73 b}$ (A) crossed with nine strains

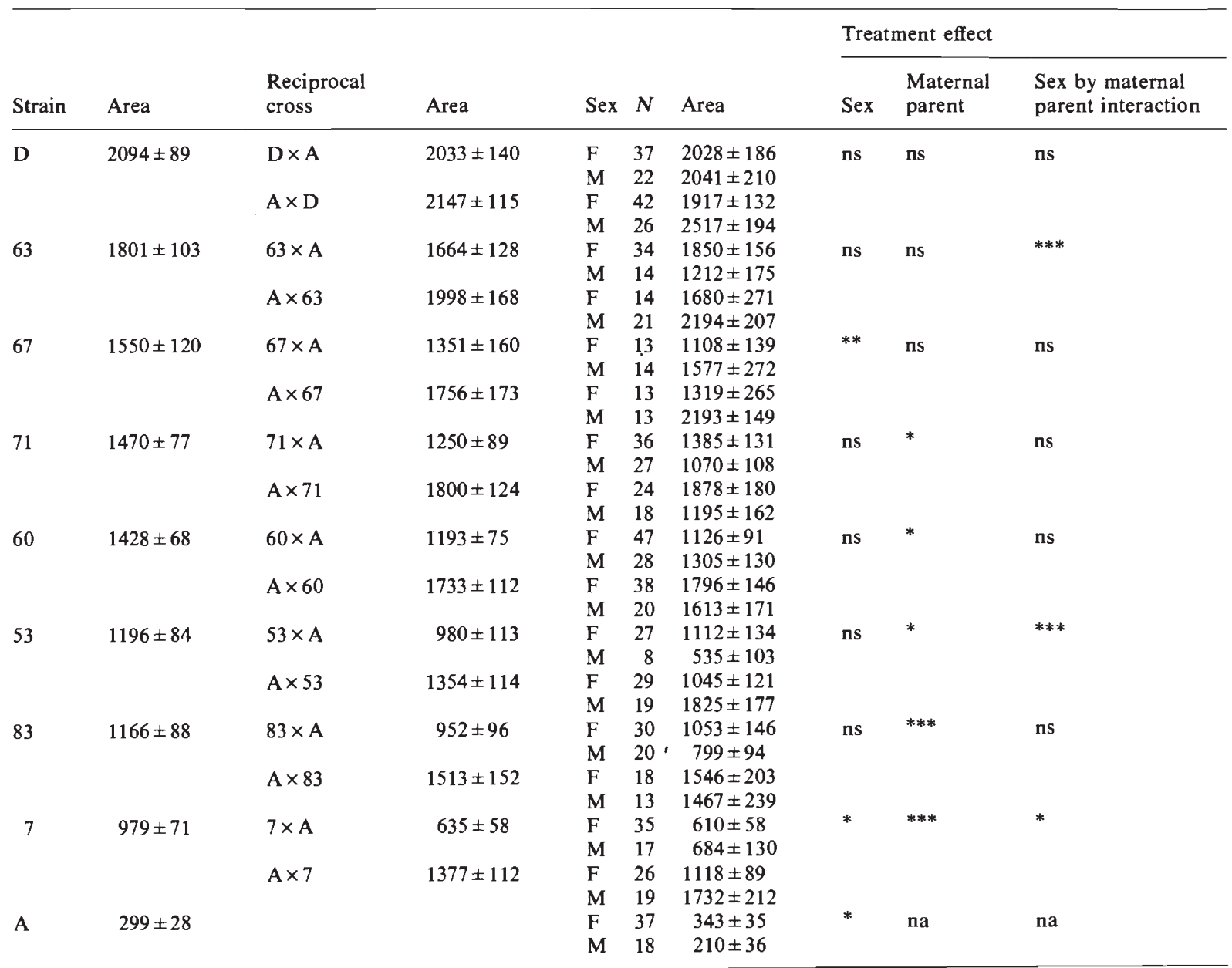

${ }^{*} P<0.05 ;{ }^{* *} P<0.01 ;{ }^{* * *} P<0.001 ;$ ns, not significant; na, not applicable.

and isogenically derived strains $(F[1,407]=63 \cdot 0$, $P<0.0001$; fig. 4) indicating maternal or sexlinked effects from $A n t p^{73 b}$ mothers in these crosses.

Differences between reciprocal female progeny were significant in progeny of $A n t p^{73 b}$ flies crossed with isogenically derived strains $60,7,83,71$ and 53 (table 1) which is consistent with the presence of highly significant maternal effects in these strains. Variation in the magnitude of the maternal effects among strains was further indicated by a significant strain by maternal parent interaction across only the female progeny of all reciprocal crosses $(F[7,483]=2.9, P<0.006)$.

Differences between sexes in the effect of an $A n t p^{73 b}$ mother (sex by maternal parent interaction) within reciprocal crosses were significant for progeny of crosses with strains 53, 63 and 7 (table
1) which is consistent with sex-linked effects in these strains (assuming maternal effects are similar for both sexes). A significant strain by maternal parent by sex interaction $(F[7,783]=2.9, \quad P<$ $0 \cdot 006)$ indicates strain differences in the magnitude of sex-linked effects.

Overall, there was a slightly larger AIII patch size in males $(1497 \pm 54$ vs. $1345 \pm 40$ square micrometres for males and females respectively, $F[1,783]=4 \cdot 3, P<0 \cdot 04)$, except for the $A n t p^{73 b}$ stock strain (table 1) where female patch size was larger. A significant cross by sex interaction $(F[8,783]=3 \cdot 1, P<0.003)$ indicates strain effects on sex differences.

The phenotypic correlation between bilateral AIII patches was $0.55(P<0.001, \mathrm{df}=646)$. The broad sense genetic correlation estimate, including all components of genetic covariation, was $1 \cdot 01$, 
indicating a high degree of common genetic variance between side 1 and side 2 patch areas. The mean asymmetry in AIII area was $75 \pm 72$ square micrometres.

Overall, 19.99 per cent of the total variance in patch size was attributable to differences among strains, $13 \cdot 21$ per cent to differences between maternal parents, $6 \cdot 25$ per cent to differences between sexes, 4.05 per cent to variation among vials within reciprocal crosses, and 56.5 per cent was due to unidentified sources.

\section{DISCUSSION}

We have shown that genetic variation pre-existing in a wild type population causes quantitative variation in the expression of an Antennapaedia phenotype. The sources of the variation are somewhat complex, since there are maternal effects, sex-linked effects, sex differences and unspecified strain differences not accounted for by the above.

The technique of examining offspring of the first generation of outcrossed Antp ${ }^{736}$ stocks puts a random half (except for linkage with $A n t p^{73 b}$ ) of a stock genetic background against half of a particular isogenically derived genetic background. The strain differences may reflect only variation in the $A n t p^{73 b}$ stock background that was crossed in with the mutation, or they could reflect subtle allelic differences at the Antennapaedia locus that may be segregating in the Dover population. Both of these sources of variation, however, are unlikely to account for much of the observed variation, since the range of strain differences in AIII patch sizes is approximately six times the mean of the original $A n t p^{73 b}$ stock. Furthermore, strain differences in the magnitude of maternal effects, sex-linked effects, and the continuous variation in patch size among crosses with isogenically derived strains all suggest more than one alleleic difference contributing to the variation among strains.

The variation observed in the AIII patch size is subtle compared to the otherwise invariant transformation of the antenna distal to AII observed in all strains, but the reliable site of the AIII patch and its anatomically continuous variability among flies may make this system useful as an assay for quantitative events underlying more discrete homeotic transformations, and for identifying potentially numerous independent factors capable of regulating expression of the Antennapaedia phenotype.
It may be possible to associate variation in specific molecular mechanisms underlying homeotic transformation with phenotypic variation in AIII patch sizes if a precise spatial and temporal focus of the $A n t p^{73 b}$ allele's effect on the AIII patch can be defined.

Rendel (1967) and others (e.g., Shrimpton and Robertson, $1988 a, b)$ have shown that the expressivity of a discrete mutant phenotype can depend on an interaction between a major gene and modifier genes in the background genotype. According to the genetic model of developmental canalization described by Rendel (1967), the variation in AIII patch size here might be viewed as a consequence of background genetic variation and environmental noise influencing a discrete anatomical site for which the homeotic mechanism controlling segmental identity is brought, by the Antp ${ }^{73 b}$ mutation, close to the threshold separating two alternative states. The AIII patch variability, therefore, may provide a sensitive measure of critical quantitative developmental events underlying homeotic transformations, despite its relatively trivial contribution to the total effect of the $A n t p^{73 b}$ mutation.

The AIII patch variation may also be useful for exploring another aspect of developmental canalization. Background quantitative genetic variation capable of potentially influencing discrete effects of major genes on canalized traits appears phenotypically "neutral" with respect to fitness as long as the phenotype remains invariant. This background genetic variation, however, is likely to represent a number of loci which may have quantitative pleiotropic effects on other traits which are expressed phenotypically and exposed to natural selection. When a discrete phenotype caused by a major gene is in an uncanalized state and expresses a variable phenotype as a result of background genetic variation, there is an opportunity, both experimentally and in natural circumstances, for genetic correlations to be detected between canalized and uncanalized traits affected by common genetic variation. This would be especially revealing in the case of homeotic traits, since such genetic correlations between quantitative measures of homeotic transformation and any other traits, if they were to be described, would identify traits whose phenotypic expression may be limited by the normally invisible constraint of being correlated to mechanisms canalizing homeotic gene function.

The variation in AIII patch size described here illustrates potential for experimentally investigating quantitative genetic regulation of homeotic gene function. 
Acknowledgements We thank the Skidmore College faculty research grant program for support, and Cindy G. Norton, Jospeh P. Hegmann, Dawson Mohler, David Domozych, John Thomas, Kevin Vaughn and Skidmore Audio-Visual Services for their assistance.

\section{REFERENCES}

CARROLL, S. B., LAYMON, R. A., MCCUTCHEON, M. A., RILEY, P. D. AND SCOTT, M. P. 1986. The localization and regulation of Antennapedia protein expression in Drosophila embryos. Cell, 47, 113-122.

CARROLL, S. B., DINARDO, S., O'FARRELL, P. H., WHITE, R. A. H. AND SCOTT, M. P. 1988. Temporal and spatial relationships between segmentation and homeotic gene expression in Drosophila embryos: Distributions of the fushi tarazu, engrailed, Sex combs reduced, Antennapedia, and Ultrabithorax proteins. Genes Dev., 2, 350-360.

GOLDSCHMIDT, E. AND LEDERMAN-KLEIN, A. 1958. Reoccurrence of a forgotten homeotic mutant in Drosophila. $J$. Heredity, 49, 262-266.

HAFEN, E., LEVINE, M. AND GEHRING, W. J. 1984. Regulation of Antennapedia transcript distribution by the Bithorax complex in Drosophila. Nature, 307, 287-289.

HOLLAND, P. W. H. 1989. Pursuing the functions of vertebrate homeobox genes: progress and prospects. Trends Neurosci., 12, 206-209.

MIYASHITA, N., LAURIE-AHLBERG, C. C., WILTON, A. N. AND EMIGH, T. H. 1986. Quantitative analysis of X Chromosome effects on the activities of the glucose 6-phosphate and 6-phosphogluconate dehydrogenases of Drosophila melanogaster. Genetics, 113, 321-335.

OUWENEEL, w. J. 1970. Genetic analysis of Loboid-Ophthalmoptera, a homeotic strain in Drosophila melanogaster. Genetica, 41, 1-20.
POSTlETHWAite, J. H. AND SCHNEIDERMAN, H. A. 1971. Pattern formation and determination in the Antenna of the homoeotic mutant Antennapedia of Drosophila melanogaster. Devl. Biol., 25, 606-640.

RENDEL, J. M. 1967. Canalisation and Gene Control. London, Logos Press.

SAS 1982. User's Guide. Cary, North Carolina, SAS Institute. SCHNEUWLY, S., KUROIWA, A. AND GEHRING, W. J. $1987 a$. Molecular analysis of the dominant homeotic Antennapedia phenotype. EMBO J., 6, 201-206.

SCHNEUWLY, S., KLEMENZ, R. AND GEHRING, W. J. $1987 b$. Redesigning the body plan of Drosophila by ectopic expression of the homeotic gene Antennapedia. Nature, $325,816-818$.

SHRIMPTON, A. E. AND ROBERTSON, A. 1988a. The isolation of polygenic factors controlling bristle score in Drosophila melanogaster. I. Allocation of third chromosome sternopleural bristle effects to chromosome sections. Genetics, $118,437-443$.

SHRIMPTON, A. E. AND ROBERTSON, A. $1988 \mathrm{~b}$. The isolation of polygenic factors controlling bristle score in Drosophila melanogaster. II. Distribution of third chromosome bristle effects within chromosome sections. Genetics, 118, 445-459.

STOCKER, R. F., EDWARDS, J. S., PALKA, J. AND SCHUBIGER, G. 1976. Projection of sensory neurons from a homeotic mutant appendage Antennapedia, in Drosophila melanogaster. Devl. Biol., 52, 210-220.

TEMPLETON, A. R. 1982. The prophecies of parthenogenesis. In Dingle, H. and Hegmann, J. P. (eds) Evolution and Genetics of Life Histories, Springer-Verlag, New York, pp. 75-102.

WADDINGTON, C. H. 1956. Genetic assimilation of the Bithorax phenotype. Evolution, 10, 1-13. 